\title{
Comparative Stability Analysis of Stator Current Error-based Estimators of Induction Motor Speed
}

Research Article

Mateusz Korzonek, Teresa Orłowska-Kowalska

Department of Electrical Machines, Drives and Measurements,

Wrocław University of Science and Technology, Wybrzeże Wyspiańskiego 27, 50-370 Wrocław, Poland

Tel: +4871320 3716, +48713202640

Received January 12, 2018; Accepted April 26, 2018

\begin{abstract}
This paper deals with the stability problem of three stator current error-based estimators of induction motor speed, especially in the regenerating operation mode. The stability of the adaptive full-order observer (AFO) and two model reference adaptive systems (MRASs) based on a stator current error (MRASCC and MRAS ${ }^{C V}$ ) is briefly analysed, and the stability borders are determined and compared. It is shown that MRAS ${ }^{\mathrm{CV}}$ speed estimator is stable in the whole operation range including the regenerating mode without any modifications. The stability enhancement method for AFO and MRAScc estimators is described, and the solution for their stability improvement is proposed. Torque-speed characteristics of the analysed MRAStype estimators in a wide range of drive speed and load torque changes are given, as well as the behaviour of estimators during transients is compared. The theoretical analysis and simulation test results are validated by experimental tests.
\end{abstract}

Keywords: induction motor • MRAS-type estimators • stability • regenerating mode

\section{Introduction}

The vector control of induction and permanent magnet synchronous motors has become an irreplaceable control technique due to the possibility of precise torque control in transient states. In particular, induction motors (IMs) are now widely used in various high-performance drive applications thanks to their advantages such as relatively low price, reliability, maintenance-free operation and the ability to work in harsh environmental conditions. The use of sensorless control additionally expands the range of applications of these drives, due to the fact that the elimination of measurement sensors in favour of state variable estimators leads to a reduction in space occupied by the drive, reduced number of cable connections, cost reduction and increased reliability (Finch and Giaouris 2008; Holtz 2006; Kazmierkowski et al. 2002; Pacas 2011; Vas 1998).

Many sensorless IM control techniques have been developed that can be generally divided into methods based on a machine mathematical model (algorithmic methods) and others that are independent of the model, such as physical methods (using natural non-linear phenomena in the machine - the so-called saliency-based techniques) or neural networks (Finch and Giaouris 2008; Holtz 2006; Pacas 2011). The most popular and often used are algorithmic methods which, based on measurements of stator winding currents and voltages and various forms of the IM mathematical model, allow estimation of stator and rotor flux as well as angular velocity and/or position of the motor shaft. The algorithmic methods can be classified into Kalman filters, state observers and simulators (Finch and Giaouris 2008; Holtz 2006; Kazmierkowski et al. 2002; Pacas 2011; Vas 1998). 
Simulators of the IM state variables can be divided into the following main groups:

- Open-loop flux estimators (the so-called voltage and current models of the rotor flux) (Holtz 2006; Kazmierkowski et al. 2002; Vas 1998)

- Estimators in which the motor speed is calculated using the slip frequency (Abu-Rub and Oikonomou 2008; Boldea et al. 2009)

- Speed estimators based on the concept of model reference adaptive systems (MRASs) (Dybkowski and Orłowska-Kowalska 2008; Kubota et al. 1993; Kumar et al. 2015; Peng and Fukao 1994; Schauder 1992; Sobczuk 1999; Tarchała et al. 2014; Verma and Chakraborty 2014)

The latter are the most popular solutions, because they are less complicated than other algorithmic methods, such as Kalman filters (Barut et al. 2007), and easy to implement in practice. These speed estimators use the estimation error of the same IM state variable calculated using two models (simulators of a given state variable): a reference model and an adaptive model, both dependent on various motor parameters (Dybkowski and Orłowska-Kowalska 2008; Kazmierkowski et al. 2002; Kubota et al. 1993; Kumar et al. 2015; Peng and Fukao 1994; Schauder 1992; Sobczuk 1999; Tarchała et al. 2014; Vas 1998; Verma and Chakraborty 2014). This error is brought to zero by an adaptation algorithm that tunes the adaptive model while simultaneously determining the motor speed.

Among many MRAS-type speed estimators, whose classification and review were made, among others in Kumar et al. (2015), the specific solutions are the speed estimators using the stator current error in the speed estimation algorithm (Dybkowski and Orłowska-Kowalska 2008; Kubota et al. 1993; Sobczuk 1999; Tarchała et al. 2014). In these systems, the IM acts as a reference model, and the adaptive models are made in a different way, depending on the selected solution. These include the MRAS ${ }^{c C}$ estimator (Dybkowski and Orłowska-Kowalska 2008) (CC represents two adaptive current models, namely stator current estimator and current model of the rotor flux), the less-known MRAS ${ }^{\mathrm{CV}}$ estimator (Sobczuk 1999) (CV represents adaptive current and voltage models, namely stator current estimator and voltage model of the rotor flux), and the most popular "the adaptive full-order observer" (AFO) (Kubota et al. 1993). All these solutions have different features, including motor parameter sensitivity; however, the most important attribute is their different stability range in the regenerating mode, especially in a low speed range.

The AFO speed estimator was often considered in the literature, including its stability analysis in a regenerating operation mode and methods of its improvement (Chen et al. 2014; Etien et al. 2010; Harnefors and Hinkkanen 2008; Hinkkanen and Luomi 2004; Kubota et al. 2002; Sunwankawin and Sangwongwanich 2006; Zaky 2011). In addition, MRAS ${ }^{C C}$ estimator has been recently analysed from the point of view of stability enhancement methods, because it has a much wider range of instability in a regenerating mode than AFO (Korzonek and OrłowskaKowalska 2016; Korzonek et al. 2017; Vonkomer and Zalman 2013). On the contrary, the stability problem of MRAS $^{\mathrm{CV}}$ estimator has never been analysed, especially in the regenerating mode. Therefore, the comparison of the stability ranges of the abovementioned three estimators is the main goal of this paper. The stability borders are determined and compared. Obtained theoretical results are verified in simulation and experimental tests.

This paper is organized as follows: In Section 2, the mathematical models of the analysed estimators are presented. Next, the stability analysis is presented for each estimator using the same methodology based on the linearization of the state estimation error equation. In Sections 3 and 4 , the simulation and experimental results are demonstrated, respectively. The paper is finished with a short conclusion in Section 5.

\section{Mathematical models of selected speed estimators}

In this section, the mathematical models of the IM and three stator current error-based IM speed estimators (AFO, MRAS ${ }^{\mathrm{CC}}$ and MRAS ${ }^{\mathrm{CV}}$ ) are presented in detail.

\subsection{Mathematical model of the IM}

The analysed angular velocity estimators are based on the mathematical model of an IM. In this paper, the IM model is presented taking into account the commonly used simplifying assumptions (such as lumped and constant parameters of the windings and symmetrical windings, and higher harmonics in the air gap, hysteresis and eddy currents have to be neglected) and using the generalised spatial vectors of the suitable state and control variables, expressed in relative units (p.u.) in a coordinate system $(x-y)$ that rotates synchronously with the rotor flux $\left(\omega_{k}=\omega_{s}\right)$ (Kazmierkowski et al. 2002; Vas 1998). The state equation of the IM electromagnetic circuits is as follows: 


$$
\begin{aligned}
& T_{N} \frac{\mathrm{d}}{\mathrm{d} t} \mathbf{x}=\mathbf{A} \mathbf{x}+\mathbf{B} \mathbf{u}_{\mathbf{s}}, \\
& \mathbf{y}=\mathbf{C x},
\end{aligned}
$$

where the state vector consists of stator current and rotor flux spatial vectors:

$$
\mathbf{x}=\left[\begin{array}{c}
\mathbf{i}_{s} \\
\psi_{r}
\end{array}\right]
$$

and the state, control and output matrices are:

$$
\mathbf{A}=\left[\begin{array}{cc}
-\frac{r_{1}}{l_{\sigma}}-\mathrm{j} \omega_{s} & \left(\frac{k_{r}}{l_{\sigma} \tau_{r}}-\mathrm{j} \frac{k_{r}}{l_{\sigma}} \omega_{m}\right) \\
r_{r} k_{r} & \left(-\frac{1}{\tau_{r}}-\mathrm{j}\left(\omega_{s}-\omega_{m}\right)\right)
\end{array}\right], \quad \mathbf{B}=\left[\begin{array}{c}
\frac{1}{l_{\sigma}} \\
0
\end{array}\right], \quad \mathbf{C}=\left[\begin{array}{ll}
1 & 0
\end{array}\right],
$$

where $\mathbf{i}_{s}, \mathbf{u}_{s}$ and $\boldsymbol{\psi}_{r}$ are the stator current, stator voltage and rotor flux spatial vectors, respectively; $k_{r}=l_{m} / l_{r}, l_{\sigma} l_{s} \sigma$, $r_{1}=r_{s}+r_{r} k_{r}^{2}, \tau_{r}=l_{r} / r_{r}, T_{N}=1 /\left(2 \pi f_{s N}\right)-$ nominal time constant; $l_{r}, l_{s}$ and $l_{m}$ are the rotor, stator and main inductance, respectively; $r_{s}$ and $r_{r}$ are the stator and rotor resistance, respectively; $\sigma$ is the total leakage factor; $\omega_{s}$ is the synchronous velocity; $\omega_{r}=\omega_{s}-\omega_{m}$ is the slip velocity; $f_{s N}$ is the nominal stator frequency.

Electromagnetic torque $m_{e}$ and equation of motion for the rotor speed $\omega_{m}$ are:

$$
\begin{aligned}
& m_{e}=\mathfrak{I}\left(\psi_{\mathbf{s}}^{*} \mathbf{i}_{\mathbf{s}}\right), \\
& \frac{\mathrm{d}}{\mathrm{d} t} \omega_{m}=\frac{1}{T_{M}}\left(m_{e}-m_{L}\right)
\end{aligned}
$$

Based on this model, different rotor flux and speed estimators can be developed. In the following subsections, mathematical models of analysed speed estimators are presented.

\subsection{Mathematical model of the AFO speed estimator}

The mathematical model of the AFO is based directly on equations (1)-(3); however, instead of measured speed, it uses the estimated one, and the additional feedback from the stator-current estimation error with the gain matrix G appears (Kubota et al. 1993):

$$
T_{N} \frac{\mathrm{d}}{\mathrm{d} t} \hat{\mathbf{x}}=\hat{\mathbf{A}}^{A F O}\left(\hat{\omega}_{m}\right) \hat{\mathbf{x}}+\mathbf{B} \mathbf{u}_{\mathbf{s}}+\mathbf{G e}_{\mathbf{i}} ;
$$

where

$$
\hat{\mathbf{A}}^{A F O}\left(\hat{\omega}_{m}\right)=\left[\begin{array}{cc}
-\frac{r_{1}}{l_{\sigma}}-\mathrm{j} \omega_{s} & \left(\frac{k_{r}}{l_{\sigma} \tau_{r}}-\mathrm{j} \frac{k_{r}}{l_{\sigma}} \hat{\omega}_{m}\right) \\
r_{r} k_{r} & \left(-\frac{1}{\tau_{r}}-\mathrm{j}\left(\omega_{s}-\hat{\omega}_{m}\right)\right)
\end{array}\right], \quad \mathbf{G}=\left[\begin{array}{l}
\mathbf{g}_{\mathbf{s}} \\
\mathbf{g}_{\mathbf{r}}
\end{array}\right],
$$

and $\mathbf{g}_{\mathbf{s}}=g_{s x}+\mathrm{j}_{s y} ; \mathbf{g}_{\mathbf{r}}=g_{r x}+\mathrm{j} g_{r y}, \mathbf{e}_{\mathbf{i}}=\mathbf{i}_{\mathbf{s}}-\hat{\mathbf{i}}_{\mathbf{s}}$ and $\mathbf{B}$ as in equation (3). 
The speed adaptation algorithm, derived using Lyapunov stability theory (Kubota et al. 1993; Vas 1998), takes the form:

$$
\frac{\mathrm{d}}{\mathrm{d} t} \hat{\omega}_{m}=K_{i} \varepsilon+K_{p} \frac{\mathrm{d}}{\mathrm{d} t} \varepsilon
$$

where the input error of the proportional integral $(\mathrm{PI})$ controller is:

$$
\varepsilon=\mathfrak{I}\left\{\mathbf{e}_{\mathbf{i}} \hat{\psi}_{\mathbf{r}}^{*}\right\}=\mathfrak{I}\left\{\left(e_{i x}+j e_{i y}\right)\left(\hat{\psi}_{r x}-j \hat{\psi}_{r y}\right)\right\}=\hat{\psi}_{r x} e_{i y}-\hat{\psi}_{r y} e_{i x},
$$

with $e_{i x, y}=i_{s x, y}-\hat{i}_{s x, y}$

\subsection{Mathematical model of the MRAS ${ }^{\mathrm{CC}}$ speed estimator}

The MRAS ${ }^{c C}$ speed estimator also uses the induction machine as a reference model (Dybkowski and OrłowskaKowalska 2008). The adaptive model consists of a current simulator model and a stator current estimator model. Both models are adjustable with the speed value estimated according to equations (7) and (8), as in the AFO estimator. The equations describing the adaptive models are as follows:

- the stator current simulator model (it is derived directly from the IM stator voltage equation by substituting the stator flux vector derivative by the rotor flux and stator current vector derivatives, taking into account the wellknown algebraic relations between the flux and current vectors (Vas 1998) and the rotor flux vector differential equation):

$$
\frac{\mathrm{d}}{\mathrm{d} t} \hat{\mathbf{i}}_{s}=\left(-\frac{r_{1}}{l_{\sigma}}-\mathrm{j} \omega_{s}\right) \hat{\mathbf{i}}_{s}+\left(\frac{k_{r}}{l_{\sigma} \tau_{r}}-\mathrm{j} \frac{k_{r}}{l_{\sigma}} \hat{\omega}_{m}\right) \hat{\boldsymbol{\psi}}_{r}+\frac{1}{l_{\sigma}} \mathbf{u}_{s},
$$

- the current model of the rotor flux:

$$
\frac{\mathrm{d}}{\mathrm{d} t} \hat{\boldsymbol{\psi}}_{r}^{i}=\left(-\frac{1}{\tau_{r}}-\mathrm{j}\left(\omega_{s}-\hat{\omega}_{m}\right)\right) \hat{\boldsymbol{\psi}}_{r}^{i}+r_{r} k_{r} \mathbf{i}_{s}
$$

Both these models depend on the estimated motor speed. The schematic diagram of this estimator is shown in Fig. 1a.

Mathematical model of the MRAS ${ }^{\mathrm{cc}}$ speed estimator can be written in a form of the state equation, similarly as AFO. It takes the form:

$$
T_{N} \frac{\mathrm{d}}{\mathrm{d} t} \hat{\mathbf{x}}=\hat{\mathbf{A}}^{C C}\left(\hat{\omega}_{m}\right) \hat{\mathbf{x}}+\mathbf{B} \mathbf{u}_{\mathbf{s}}+\mathbf{G e}_{\mathbf{i}}+\mathbf{K}_{C C} \mathbf{y}
$$

where $\mathbf{B}$ is as in equation (3) and:

$$
\hat{\mathbf{A}}^{C C}\left(\hat{\omega}_{m}\right)=\left[\begin{array}{cc}
-\frac{r_{1}}{l_{\sigma}}-\mathrm{j} \omega_{s} & \frac{k_{r}}{l_{\sigma} \tau_{r}}-\mathrm{j} \frac{k_{r}}{l_{\sigma}} \hat{\omega}_{m} \\
0 & -\frac{1}{\tau_{r}}-\mathrm{j}\left(\omega_{s}-\hat{\omega}_{m}\right)
\end{array}\right] \quad \mathbf{K}_{C C}=\left[\begin{array}{cc}
0 & 0 \\
r_{r} k_{r} & 0
\end{array}\right],
$$



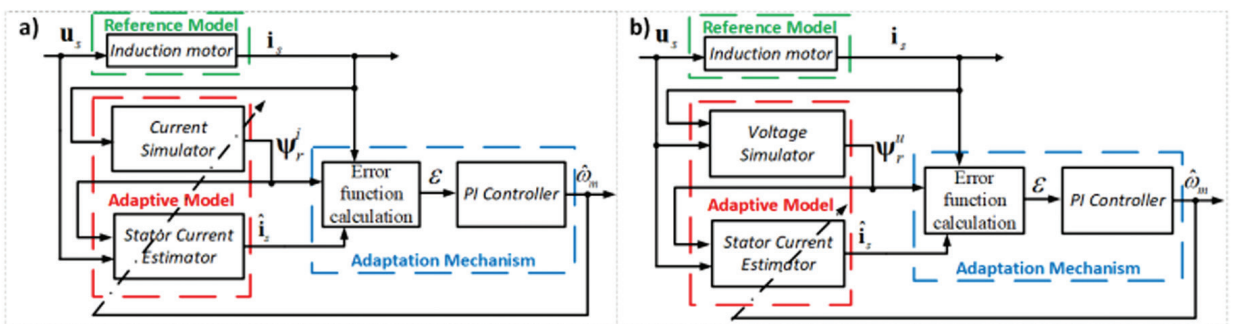

Fig. 1. The block diagrams of selected speed estimators: a) MRAS ${ }^{c c}$, b) MRAS ${ }^{c v}$

\subsection{Mathematical model of the MRAS ${ }^{\mathrm{CV}}$ speed estimator}

The third speed estimator analysed in this paper is the MRAS ${ }^{C V}$ estimator (Sobczuk 1999), whose block diagram is shown in Fig. 1b. It differs from the MRAS ${ }^{\mathrm{cC}}$ estimator, in that instead of the adapted current flux simulator, an un-adapted voltage flux model (independent of the rotor speed) was used (it is derived by rearranging the IM stator winding equation using directly the algebraic relations between the stator/rotor flux space vectors and stator/rotor current vectors; Vas 1998):

$$
\frac{\mathrm{d}}{\mathrm{dt}} \hat{\boldsymbol{\psi}}_{r}^{u}=-\mathrm{j} \omega_{s} \hat{\psi}_{r}^{u}+\left(-\frac{r_{s}}{k_{r}}-T_{N} \frac{l_{\sigma}}{k_{r}} \frac{\mathrm{d}}{\mathrm{dt}}-\mathrm{j} \frac{l_{\sigma}}{k_{r}} \omega_{s}\right) \mathbf{i}_{s}+\frac{1}{k_{r}} \mathbf{u}_{s}
$$

Mathematical model of the MRAS ${ }^{\mathrm{CV}}$ speed estimator can be written in the form of state equation, similarly as $M_{R A S}{ }^{C C}$ and AFO. It takes the form:

$$
T_{N} \frac{\mathrm{d}}{\mathrm{dt}} \hat{\mathbf{x}}=\hat{\mathbf{A}}^{C V}\left(\hat{\omega}_{m}\right) \hat{\mathbf{x}}+\mathbf{B}_{C V} \mathbf{u}_{\mathbf{s}}+\mathbf{G e}_{\mathbf{i}}+\mathbf{K}_{C V} \mathbf{y},
$$

where

$$
\hat{\mathbf{A}}^{C V}\left(\hat{\omega}_{m}\right)=\left[\begin{array}{cc}
-\frac{r_{1}}{l_{\sigma}}-\mathrm{j} \omega_{s} & \frac{k_{r}}{l_{\sigma} \tau_{r}}-\mathrm{j} \frac{k_{r}}{l_{\sigma}} \hat{\omega}_{m} \\
0 & -\mathrm{j} \omega_{s}
\end{array}\right] ; \mathbf{B}_{C V}=\left[\begin{array}{c}
\frac{1}{l_{\sigma}} \\
\frac{1}{k_{r}}
\end{array}\right] ; \mathbf{K}_{C V}=\left[\begin{array}{cc}
0 & 0 \\
-\frac{r_{s}}{k_{r}}-T_{N} \frac{l_{\sigma}}{k_{r}} \frac{\mathrm{d}}{\mathrm{dt}}-\mathrm{j} \frac{l_{\sigma}}{k_{r}} \omega_{s} & 0
\end{array}\right] .
$$

The speed value is estimated according to equations (7) and (8), as in the AFO and MRAS ${ }^{c c}$ estimator.

According to the mathematical models presented earlier, the generalised block diagram of the analysed speed estimators can be drawn, as shown in Fig. 2.

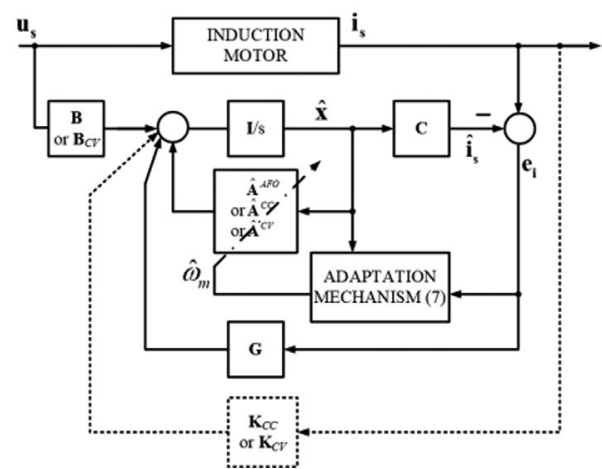

Fig. 2. The generalized block diagram of $A F O, M R A S^{C C}$ and MRAS ${ }^{C V}$ speed estimators (matrix $\mathbf{K}_{C C}$ or $\mathbf{K}_{C V}$ and $\mathbf{B}_{C V}$ appear only in $M R A S^{C C}$ or MRAS respectively) 


\section{Stability analysis of selected speed estimators}

This section deals with the detailed stability analysis of AFO, MRAS ${ }^{\mathrm{CC}}$ and MRAS ${ }^{\mathrm{CV}}$. First, two approaches for stability improvement are described. Next, the main steps of stability analysis methodology are presented. At the end of this section, a detailed analysis of selected speed estimators and the method used to improve their stability is presented.

\subsection{General remarks}

Previous research showed that almost all known MRAS-type speed estimators are unstable in a certain range of regenerating mode (Bensiali et al. 2015; Korzonek and Orłowska-Kowalska 2016; Korzonek et al. 2017; Pal et al. in press; Purti et al. 2015; Rashed et al. 2003; Stoicuta and Pana 2016; Verma and Chakraborty 2014; Vonkomer and Zalman 2013). In addition, AFO with matrix $\mathbf{G}=0$ has some unstable operating points in this mode (Chen et al. 2014; Etien et al. 2010; Harnefors and Hinkkanen 2008; Hinkkanen and Luomi 2004; Kubota et al. 2002; Sunwankawin and Sangwongwanich 2006; Zaky 2011). Therefore, research on the stabilization methods of the estimators has been undertaken. So far, two approaches have been proposed:

1 The proper selection of the gain matrix $\mathbf{G}$ elements (Chen et al. 2014; Etien et al. 2010; Harnefors and Hinkkanen 2008; Hinkkanen and Luomi 2004; Kubota et al. 2002; Sunwankawin and Sangwongwanich 2006; Zaky 2011)

2 The modification of the input error (equation 8) of the speed adaptation algorithm (equation 7 ) to the following form, proposed first in Hinkkanen and Luomi (2004):

$$
\begin{aligned}
\mathcal{E} & =\mathfrak{I}\left\{e^{-j \varphi} \mathbf{e}_{\mathbf{i}} \psi_{\mathbf{r}}^{*}\right\}=\mathfrak{I}\left\{(\cos \varphi-\mathrm{j} \sin \varphi)\left(e_{i x}+\mathrm{j} e_{i y}\right)\left(\hat{\psi}_{r x}-\mathrm{j} \hat{\psi}_{r y}\right)\right\}=, \\
& =\hat{\psi}_{r x} e_{i y} \cos \varphi-\hat{\psi}_{r x} e_{i x} \sin \varphi-\hat{\psi}_{r y} e_{i x} \cos \varphi-\hat{\psi}_{r y} e_{i x} \sin \varphi
\end{aligned}
$$

which introduces a certain angle shift $\varphi$ between the stator current error and the rotor flux vector. Both methods enable the minimisation of the unstable region of the estimator to the line $\omega_{s}=0$.

However, the first method is more difficult in practical implementation due to the fact that it requires calculation of at least four $g_{i j}$ parameters, while in the second method it is enough to calculate one parameter $\varphi$ (Etien et al. 2010; Harnefors and Hinkkanen 2008; Hinkkanen and Luomi 2004; Korzonek and Orłowska-Kowalska 2016; Korzonek et al. 2017; Vonkomer and Zalman 2013). Thus, in this paper, only the second method of estimator stabilisation is analysed.

To conduct a stability analysis of the speed estimators, the five steps of the following algorithm should be realised (Korzonek et al. 2017):

- Defining the full estimator model, i.e. extending the estimator state equations $(5,11,14)$ by the adaptation mechanism (equation 7 ) with proper expression for $\varepsilon$.

- Calculating the state estimation error equation, obtained by subtracting the extended state equation of the estimator from state equation of the IM model (equations 1-4), where the error state vector is defined as follows:

$$
\mathbf{e}=\left[\begin{array}{c}
\mathbf{e}_{\mathbf{i}} \\
\mathbf{e}_{\boldsymbol{\psi}} \\
\mathrm{e}_{\omega}
\end{array}\right]=\left[\begin{array}{c}
\mathbf{i}_{s}-\hat{\mathbf{i}}_{s} \\
\boldsymbol{\psi}_{r}-\hat{\psi}_{r} \\
\omega_{m}-\hat{\omega}_{m}
\end{array}\right] .
$$

- Linearization of the extended non-linear state equation of the estimation error around a fixed operating point:

$$
\mathbf{e}=\mathbf{e}_{0}+\Delta \mathbf{e} ; \quad \hat{\omega}_{m}=\hat{\omega}_{m 0}+\Delta \hat{\omega}_{m}
$$


and obtaining the following equation:

$$
T_{N} \frac{\mathrm{d}}{\mathrm{d} t} \Delta \mathbf{e}_{0}=\hat{\mathbf{A}}_{0}^{e} \Delta \mathbf{e}_{0}+\Delta \hat{\mathbf{A}}_{0}^{e} \mathbf{e}_{0} ;
$$

where upper index $e$ concerns the chosen estimator type.

- Calculation of the eigenvalues of the linearized state matrix $\hat{\mathbf{A}}_{0}^{e}$ (poles of the estimator) based on the estimator characteristic equation:

$$
\operatorname{det}\left(s \mathbf{I}-\hat{\mathbf{A}}_{0}^{e}\right)=0 .
$$

- Analysis of the sign of the real parts of all the estimator poles at fixed operating points - a positive real part of any pole indicates an unstable operating point.

- Determination of instability boundaries by comparing the value of the determinant of a linearized state matrix to zero:

$$
\operatorname{det} \hat{\mathbf{A}}_{0}^{e}=0 \text {. }
$$

\subsection{Stability analysis of AFO speed estimator}

The detailed stability analysis of AFO estimator is given in Etien et al. (2010) and Korzonek et al. (2017), using the methodology described earlier. After obtaining the full mathematical model of this estimator (including the speed adaptation algorithm (equations 7 and 8 ) and linearization, its state estimation error equation around an arbitrary operating point, the following linearized state matrix is obtained:

$$
\hat{\mathbf{A}}_{0}^{A F O}=\left[\begin{array}{ccc}
-\frac{r_{1}}{l_{\sigma}}-\mathrm{j} \omega_{s 0}-\mathbf{g}_{s} & \frac{k_{r}}{l_{\sigma} \tau_{r}}-\mathrm{j} \frac{k_{r}}{l_{\sigma}} \hat{\omega}_{m 0} & -\mathrm{j} \frac{k_{r}}{l_{\sigma}} \boldsymbol{\psi}_{r 0} \\
r_{r} k_{r}-\mathbf{g}_{r} & -\frac{1}{\tau_{r}}-\mathrm{j}\left(\omega_{s 0}-\hat{\omega}_{m 0}\right) & \mathrm{j} \boldsymbol{\psi}_{r 0} \\
\mathbf{a}_{s O} & \mathbf{a}_{r O} & a_{\omega O}
\end{array}\right],
$$

where $\omega_{s 0}-\hat{\omega}_{m 0}=\hat{\omega}_{r 0} ; \mathbf{a}_{s 0}=\left[\begin{array}{ll}a_{51} & a_{52}\end{array}\right] ; \mathbf{a}_{r 0}=\left[\begin{array}{ll}a_{53} & a_{54}\end{array}\right] ; a_{\omega 0}=a_{55}$.

Details on values of the $a_{51}-a_{55}$ coefficients and the matrix $\Delta \hat{\mathbf{A}}_{0}^{A F O}$ (equation 19) are given in Appendix. It is also assumed that $\psi_{r x 0}=\psi_{r e f}, \psi_{r y 0}=0$.

Assuming $\mathbf{g}_{s}=\mathbf{0}, \mathbf{g}_{r}=\mathbf{0}, \varphi=0, K_{p}=0$, the value of the state matrix determinant is calculated as follows:

$$
\operatorname{det} \hat{\mathbf{A}}_{0}^{A F O}=-\frac{k_{r} K_{i} \Psi_{r e f}^{2}}{l_{\sigma}^{2} l_{r}} \omega_{s 0}\left(l_{r} r_{s} \omega_{r 0}+l_{r} r_{r} k_{r}^{2} \omega_{s 0}+l_{\sigma} r_{r} \omega_{s 0}\right),
$$

which is equal to zero, when

$$
\omega_{s 0}=0 \text { or } \omega_{s 0}=\omega_{m 0} \frac{r_{s}}{r_{s}+\frac{l_{\sigma}}{\tau_{r}}+r_{r} k_{r}^{2}} .
$$

The two expressions obtained in equation (24) constitute two lines, D1 and D2 ${ }^{\mathrm{AFO}}$, which determine the borders of the estimator unstable operation points. They show the relation $\omega_{s 0}=f\left(\omega_{m 0}\right)$, but can be recalculated to obtain the relation between the torque and speed, i.e. $m_{L}=f\left(\omega_{m 0}\right)$, according to equation (25) (Korzonek et al. 2017): 


$$
\left\{\begin{array}{c}
m_{L}=-\frac{\Psi_{r e f}^{2}}{r_{r}} \omega_{m 0} \\
m_{L}=-\frac{\Psi_{r e f}^{2}}{r_{r}} \omega_{m 0}\left(\frac{\frac{l_{\sigma}}{\tau_{r}}+r_{r} k_{r}^{2}}{r_{s}+\frac{l_{\sigma}}{\tau_{r}}+r_{r} k_{r}^{2}}\right)
\end{array} \rightarrow \mathrm{D} 1\right.
$$

These lines are drawn in Fig. 3a in the final part of Section 3.

\subsection{Stability analysis of MRAS ${ }^{C C}$ speed estimator}

The detailed stability analysis of MRAS ${ }^{\mathrm{CC}}$ estimator is presented in Korzonek and Orłowska-Kowalska (2016) and Korzonek et al. (2017). After obtaining the full mathematical model of this estimator and linearization, its state estimation error equation in an arbitrary operating point, the following linearized state matrix is obtained:

$$
\hat{\mathbf{A}}_{0}^{C C}=\left[\begin{array}{ccc}
-\frac{r_{1}}{l_{\sigma}}-\mathrm{j} \omega_{s 0}-\mathbf{g}_{s} & \frac{k_{r}}{l_{\sigma} \tau_{r}}-\mathrm{j} \frac{k_{r}}{l_{\sigma}} \hat{\omega}_{m 0} & -\mathrm{j} \frac{k_{r}}{l_{\sigma}} \boldsymbol{\psi}_{r 0} \\
-\mathbf{g}_{r} & -\frac{1}{\tau_{r}}-\mathrm{j}\left(\omega_{s 0}-\hat{\omega}_{m 0}\right) & \mathrm{j} \boldsymbol{\psi}_{r 0} \\
\mathbf{a}_{s O} & \mathbf{a}_{r O} & a_{\omega O}
\end{array}\right],
$$

where $\omega_{s 0}-\hat{\omega}_{m 0}=\hat{\omega}_{r 0} ; \mathbf{a}_{s 0}=\left[\begin{array}{ll}a_{51} & a_{52}\end{array}\right] ; \mathbf{a}_{r 0}=\left[\begin{array}{ll}a_{53} & a_{54}\end{array}\right] ; a_{\omega 0}=a_{55}$ and $a_{51}-a_{55}$ coefficients as well as the matrix $\Delta \hat{\mathbf{A}}_{0}^{C C}$ (equation 19) are the same as for AFO.

Similarly, assuming $\mathbf{g}_{s}=\mathbf{0}, \mathbf{g}_{r}=\mathbf{0}, \varphi=0, K_{p}=0$, the value of the state matrix determinant is calculated as follows:

$$
\operatorname{det} \hat{\mathbf{A}}_{0}^{C C}=-\frac{k_{r} K_{i} \Psi_{r e f}^{2}}{l_{\sigma}^{2} l_{r}} \omega_{s 0}\left(l_{r} r_{s} \omega_{r 0}+l_{r} r_{r} k_{r}^{2} \omega_{r 0}+l_{\sigma} r_{r} \omega_{s 0}\right),
$$

which is equal to zero, when

$$
\omega_{s 0}=0 \text { or } \omega_{s 0}=\omega_{m 0} \frac{r_{s}+r_{r} k_{r}^{2}}{r_{s}+\frac{l_{\sigma}}{\tau_{r}}+r_{r} k_{r}^{2}}
$$

The two expressions obtained in equation (28) constitute two lines, D1 and D2 ${ }^{\mathrm{CC}}$, which determine the MRAS ${ }^{\mathrm{CC}}$ instability borders:

$$
\left\{\begin{array}{c}
m_{L}=-\frac{\Psi_{r e f}^{2}}{r_{r}} \omega_{m 0} \\
m_{L}=-\frac{\Psi_{r e f}^{2}}{r_{r}} \omega_{m 0}\left(\frac{\frac{l_{\sigma}}{\tau_{r}}}{r_{s}+\frac{l_{\sigma}}{\tau_{r}}+r_{r} k_{r}^{2}}\right)
\end{array} \rightarrow \mathrm{D} 2^{C C}\right.
$$

These lines are drawn in Fig. $3 b$ in the final part of Section 3. 


\subsection{Stability analysis of MRAS ${ }^{c v}$ speed estimator}

To properly analyse the stability of the MRAS ${ }^{\mathrm{CV}}$ estimator, the basic IM mathematical model (equations 1-4) has to be rearranged. The second state equation in (1a), describing the rotor flux vector dynamics, must be presented using the voltage, not the current flux model. Next, the state estimation error equation, obtained by subtracting the extended state equation of this estimator (equations 14 and 15) from the rearranged state equation of the IM model, is received. After linearization, the following state matrix is obtained:

$$
\hat{\mathbf{A}}_{0}^{C V}\left(\hat{\omega}_{m}\right)=\left[\begin{array}{ccc}
-\frac{r_{1}}{l_{\sigma}}-\mathrm{j} \omega_{s 0} & \frac{k_{r}}{l_{\sigma} \tau_{r}}-\mathrm{j} \frac{k_{r}}{l_{\sigma}} \hat{\omega}_{m 0} & -\mathrm{j} \frac{k_{r}}{l_{\sigma}} \Psi_{r 0} \\
0 & -\mathrm{j} \omega_{s 0} & 0 \\
\mathbf{a}_{s O} & \mathbf{a}_{r O} & a_{\omega O}
\end{array}\right] .
$$

where $\omega_{s 0}-\hat{\omega}_{m 0}=\hat{\omega}_{r 0} ; \mathbf{a}_{s 0}=\left[\begin{array}{ll}a_{51} & a_{52}\end{array}\right] ; \mathbf{a}_{r 0}=\left[\begin{array}{ll}a_{53} & a_{54}\end{array}\right] ; a_{\omega 0}=a_{55}$ and $a_{51}-a_{55}$ coefficients (equation 19) are the same as for AFO and MRAS ${ }^{c C}$ as well the matrix $\Delta \hat{\mathbf{A}}_{0}^{C V}$ is almost the same.

Assuming, as in previous cases, that $\mathbf{g}_{s}=\mathbf{0}, \mathbf{g}_{r}=\mathbf{0}, \varphi=0, K_{p}=0$, the value of the state matrix determinant is calculated as follows:

$$
\operatorname{det} \hat{\mathbf{A}}_{0}^{C V}\left(\hat{\omega}_{m}\right)=-K_{i} \psi_{r e f}^{2} \omega_{s 0}^{2} \frac{r_{1}}{l_{\sigma}} \frac{k_{r}}{\tau_{r}}
$$

which is equal to zero, when

$$
\omega_{s 0}=0 \text {, }
$$

which means that unstable operating points of this estimator lay only on the line $\omega_{s 0}=0$ (line D1), and this estimator is stable as well in the motoring as in the regenerating modes, on the contrary to the others known estimators of the IM speed.

In Fig. 3, the comparison of the stability borders of three analysed speed estimators is shown. It can be noted at presented diagrams, that in the generating mode, the AFO and MRAS ${ }^{\mathrm{CC}}$ estimators behave unstably (as it results from equations [25] and [29]), as opposed to the MRAS ${ }^{\mathrm{CV}}$ estimator, which is stable in the whole operation region, except of $\omega_{s 0}=0$. Moreover, it should be highlighted that parameters $K_{p}, K_{i}$ in the adaptation mechanism (7) do not influence the positions of the lines D1 and D2.
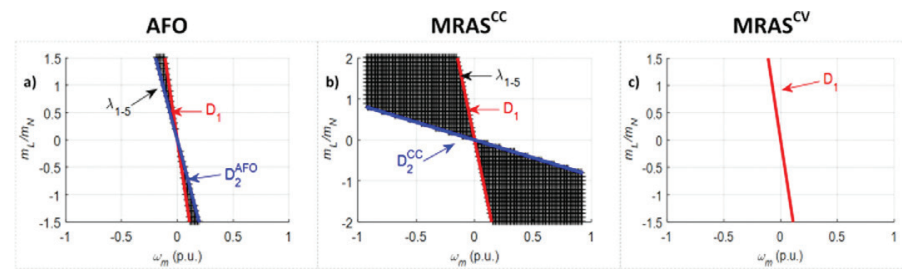

Fig. 3. Theoretical analysis of the stability borders of speed estimators: a) AFO, b) MRAS'c , c) MRAS ${ }^{\mathrm{cv}}$ for $K_{p}=1, K_{i}=30 T_{N}$

\subsection{Stability enhancement method for AFO and MRAS ${ }^{C C}$ speed estimators}

As mentioned in Section 3.1, it is possible to improve the stability of AFO and MRAS ${ }^{c c}$ estimators, using the modified adaptation algorithm (equation 16). Based on a stability analysis of these estimators with the modified adaptation algorithm, the following value of the shift angle was proposed in Korzonek et al. (2017):

$$
\varphi=\tan ^{-1}\left(\frac{l_{r}}{r_{r}} \omega_{m 0}\right),
$$

and is the same for AFO and MRAS ${ }^{\mathrm{cC}}$. 
Fig. 4 shows the unstable operating points when equation (33) is applied in AFO (Fig. 4a) and MRAScc (Fig. 4b). In Fig. 4c, the unstable operating points for the MRAS ${ }^{\mathrm{CV}}$ without any modification are demonstrated for a comparison. The unstable region in the regenerating mode of AFO and MRAS ${ }^{\mathrm{CC}}$ disappeared; however, they appeared in the motoring mode. This is the disadvantage of the shift angle improvement method, but it is simple to solve. The value of the shift angle should be modified from (33) to 0 or in the opposite direction, when the IM changes the operating mode from regenerating to motoring mode and vice versa.
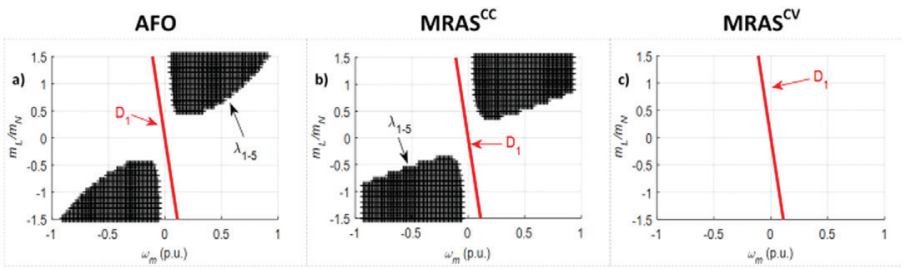

Fig. 4. Theoretical results with the stability borders obtained by the adaptation rule modification for chosen speed estimators: a) AFO, b) MRAS ${ }^{\circ C}$, c) MRASCr (without any modification) for $K_{p}=1, K_{i}=30 T_{N}$

\section{Simulation and experimental test of the speed estimators}

This section is divided into four subsections. In the first one, the description of simulation research and the experimental test bench are presented. In the next subsections, the simulation and the experimental results of analysed estimators in open-loop operation, closed-loop operation and under parameter changes are demonstrated and discussed.

\subsection{Short description of the control structure and testing scenarios}

Simulation and experimental results of studies related to the stability of these estimators presented in this section were obtained using the Direct Rotor Field-Oriented Control (DRFOC) structure, as shown in Fig. 5. This control scheme was designed in the Matlab/Simulink program, in which the analysed estimators worked in an open-loop system (outside the feedback loop from the actual motor speed) to test their stability performance. The experimental stand consists of two IMs (1.1 kW and $1.5 \mathrm{~kW})$, two voltage converters, measuring sensors (three sensors for current measurement, a sensor for measuring the DC bus voltage $\left(u_{D C}\right)$, an incremental encoder for measuring the motor speed) and a system for rapid prototyping (dSpace 1103). The rated motor parameters and equivalent circuit parameters are summarized in Table 1.

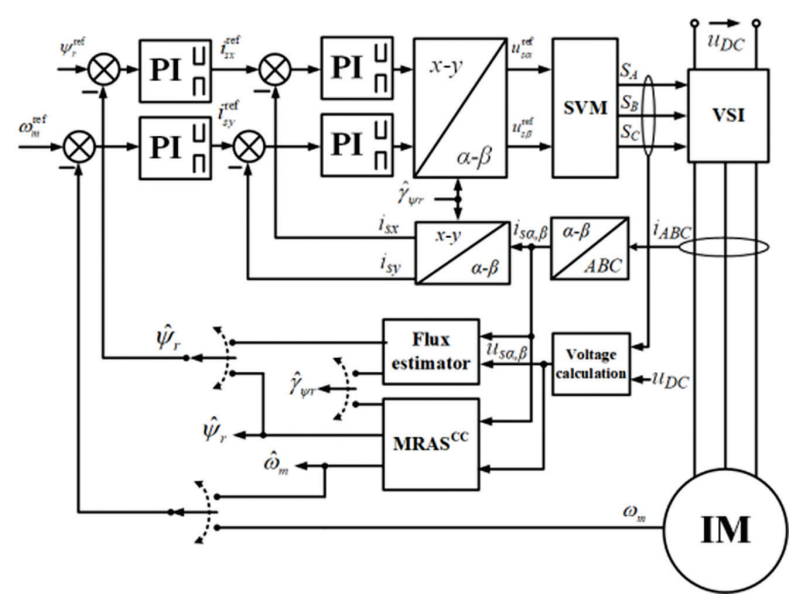

Fig. 5. Block diagram of the DFOC structure for the IM with MRAS estimators

Fig. 6 shows the exemplary transients of the motor speed under load torque changes for two different speed reference values: $\omega_{m}=0.1 \omega_{m N}$ and $\omega_{m}=0.7 \omega_{m N}$, respectively. Based on these transients, the "torque-speed" characteristics were created for the analysed speed estimators. 
Table 1. Parameters of the tested IM

\begin{tabular}{lcc}
\hline Parameter & Value (physical unit) & Value (per unit) \\
\hline \hline Nominal power & $P_{N}=1100(\mathrm{~W})$ & $p_{N}=0.6377$ \\
Nominal torque & $M_{N}=7.557(\mathrm{~N})$ & $m_{N}=0.6881$ \\
Nominal voltage & $U_{N}=230(\mathrm{~V})$ & $u_{N}=0.7071$ \\
Nominal current & $I_{N}=2.5(\mathrm{~A})$ & $i_{N}=0.7071$ \\
Nominal motor speed & $n_{N}=1390(\mathrm{rpm})$ & $\omega_{m N}=0.9267$ \\
Magnetizing inductance & $L_{m}=426.6(\mathrm{mH})$ & $l_{m}=1.4499$ \\
Stator/rotor inductance & $L_{s}=L_{r}=398.38(\mathrm{mH})$ & $l_{r}=l_{s}=1.5394$ \\
Stator resistance & $R_{s}=5.019(\Omega)$ & $r_{s}=0.0546$ \\
Rotor resistance & $\mathrm{R}_{r}=6.497(\Omega)$ & $r_{r}=0.0706$ \\
Flux & $\psi_{r}=0.8428(\mathrm{~Wb})$ & $\psi_{r}=0.8141$ \\
Frequency & $f_{s N}=50(\mathrm{~Hz})$ & - \\
The number of pole pairs & $p_{b}=2$ & - \\
\hline
\end{tabular}

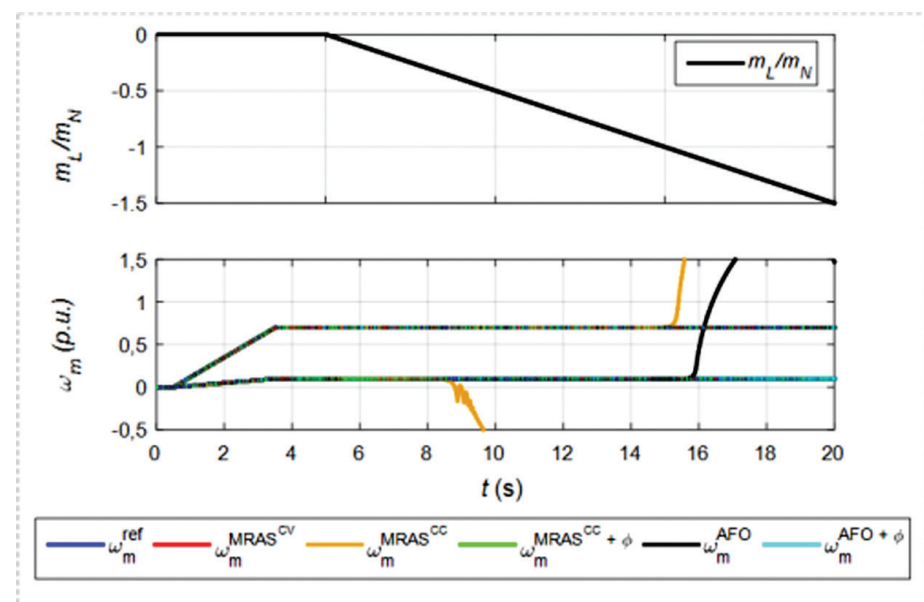

Fig. 6. Simulation results. Sample trajectories of (a) load torque and (b) speed reference and speed obtained from different estimators

The tests' scenario was as follows: first, from 0.5-3.5 seconds the reference speed was changed linearly to a set value; within 5 seconds the measured and estimated speeds reached this set value. In the fifth second, the load torque (regenerating mode) was activated, which for the next 15 seconds was increased from zero to one (p.u.) and finally was $50 \%$ greater than its nominal value $\left(1.5 m_{L N}\right)$. The whole process ended after 20 seconds. In the presented sample transients (Fig. 6b), it can be seen that in the generating range, the AFO and MRAS ${ }^{\mathrm{CC}}$ estimators, in which the stability improvement method is not applied, behave unstably, as opposed to the MRAS ${ }^{\mathrm{CV}}$ estimator. However, the MRAS ${ }^{c C}$ becomes unstable much faster (for a smaller load torque value) than the AFO, which confirms the theoretical analysis (Fig. 3a,b).

\subsection{Torque-speed characteristics}

The simulation and experimental results in a form of torque-speed characteristics of each of the analysed estimators (without or with the stability improvement method based on a shift angle $\varphi$ for AFO and MRAS ${ }^{c C}$ ) are shown in Fig. 7. The first column shows the simulation results, while the experimental results are demonstrated in the second column. Black lines show different reference values of the speed regarding the load torque changes, while the green lines show the characteristic of the speed estimator. If the estimator is stable, then the green line covers the black one; if the estimator becomes unstable, the green line differs from the black one significantly. During the analysis, the coefficients of the adaptation mechanism were, respectively, $K_{p}=1$ and $K_{i}=30 T_{N^{*}}$.

As the theoretical analysis shows (Fig. 3c), the MRAS ${ }^{\mathrm{CV}}$ estimator is stable in almost the entire operating range, except for the straight line D1 (Fig. 7, the last row), contrary to AFO and MRAS ${ }^{C C}$ with classical adaptation mechanism (equation 6). In the case of the MRAS ${ }^{\mathrm{CC}}$ estimator, the range of unstable operating points covers the 
greater part of the regenerating mode compared to AFO (Fig. 3a and b and Fig. 7 rows I and III), which is associated with worse properties of this estimator in comparison to AFO estimator. However, after applying the appropriate method to improve the estimator stability, using equation (16) with shift angle $\varphi$ given by equation (33) for the MRAS $^{\mathrm{CC}}$ and AFO estimators, this unstable operating range can be compensated only for the straight line D1 (Fig. 4a and b and Fig. 7, rows II and IV). The disadvantage of this method is the appearance of unstable operating points in the motoring operation mode (dotted area in Fig. 4a and b). As it was said before, this negative feature of MRAS ${ }^{c C}$ and AFO estimators can be eliminated by switching the value of the angle $\varphi$ from value $(33)$ to 0 , when drive is changing the operation from regenerating to motoring mode and vice versa.

The simulation results (Fig. 7a in rows II and IV) confirm all the discussed properties of the analyzed estimators. For the AFO and MRAS ${ }^{\mathrm{CC}}$, with the proposed stability improvement method, the angle $\varphi$ value was changed to zero when switching from the regenerating operation to motoring mode and vice versa, which was marked on the graphs.

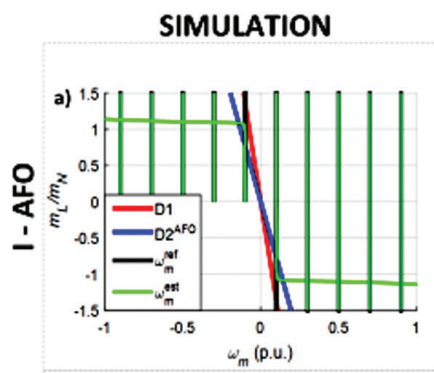

\section{EXPERIMENT}
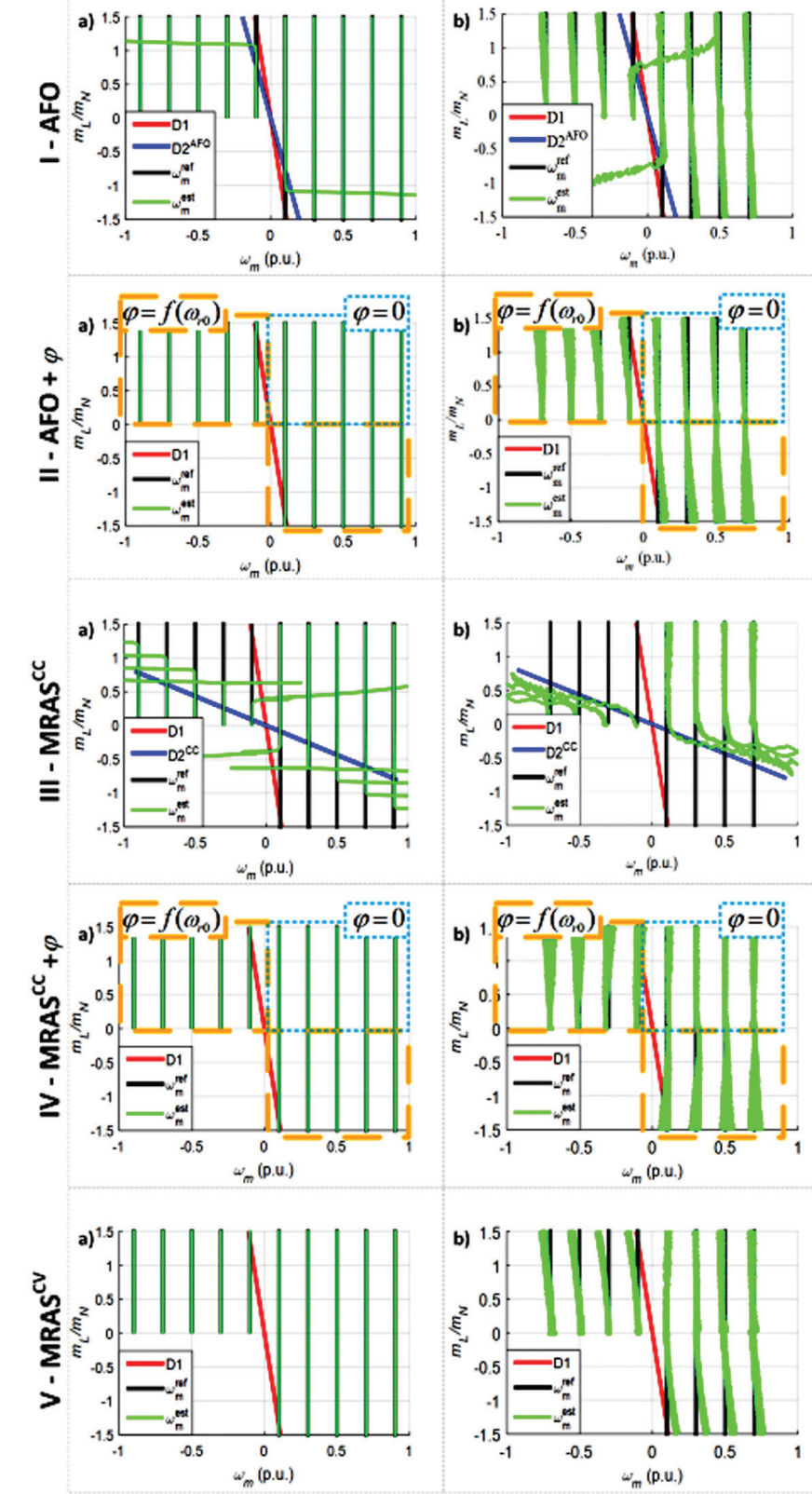

Fig. 7. Torque-speed characteristics. Simulation (column a) and experimental results (column b) of the analysed estimators 
The experimental results (Fig. 7b, second column) also confirm all the discussed properties of the estimators. It is worth noting that for MRAS ${ }^{\mathrm{CV}}$ working in the regenerating mode of the drive system, along with the increase in the load torque, the speed estimation error occurs, and for the MRAS ${ }^{\mathrm{CC}}$ with the modified adaptation algorithm, larger oscillations appear. This feature has not been observed in simulation tests, where the estimator parameters were the same as the IM. Thus, it can be caused by the influence of real motor parameter changes during the drive system operation, while in the mathematical models of estimators the nominal parameters were used. It shows that these estimators are sensitive to motor parameter changes (or parameter identification errors), however in different way.

\subsection{Influence of parameter identification errors on the estimators' stability}

The influence of four parameter changes (stator and rotor resistance, stator and rotor leakage inductance) on the stability properties of MRAS ${ }^{\mathrm{CC}}, \mathrm{MRAS}^{\mathrm{CV}}$ and AFO for three different reference speed values was analysed in Orłowska-Kowalska and Dybkowski (2010). All estimators are not sensitive to rotor resistance variation, but the other motor parameters have impact on the stability of AFO and MRAS ${ }^{c V}$. Unfortunately, the worst stability under parameter mismatch has the MRAS ${ }^{\mathrm{cV}}$ estimator. It is worth noting that the MRAS ${ }^{\mathrm{CC}}$ is stable for all parameter changes from $50 \%$ to $150 \%$ of their nominal values.

In Korzonek and Orłowska-Kowalska (2017a) and Korzonek and Orłowska-Kowalska (2017b), the influence of three main parameter mismatch (rotor and stator resistance and main inductance) on the stability range of AFO and MRAS ${ }^{C C}$ with application of the adaptation rule modification (equations (16) and (33), respectively) was analysed. In both cases, the stability improvement method is robust to the rotor resistance mismatch only, but the other two parameters have the noticeable impact on the estimation accuracy only in a low speed region, also in the regenerating mode. The authors concluded that the best way for improvement in the estimation quality in the mentioned region will be the application of simple stator resistance and main inductance estimators, which is now under consideration and is out of scope of this paper.

\subsection{Closed-loop operation}

The behaviour of the analysed stator current error-based estimators, with or without stability improvement method, was tested in simulation in the closed-loop operation of the DRFOC structure (Fig. 5). The load torque was changed as shown in Fig. 6, and the reference speed value was set to $\omega_{m}{ }^{r e f}=0.1 \omega_{m N^{*}}$

When AFO and MRAS ${ }^{\mathrm{CC}}$ without the stability range enhancement method reach the unstable zones, the drive operation can be similar to the one shown in Fig. 8 (row I) and Fig. 8 (row III), respectively. The real values of the motor speed and the rotor flux change significantly. Because the speed estimator, which normally loses its stability in the regenerating mode for some value of the load torque (Fig. 6), is operating in the closed loop, it follows the reference speed value due to forcing the motor state (especially the rotor flux value), which destabilize the real motor speed, as shown in Fig. 8, rows I and III, respectively. Especially, the rotor flux reaches very high values, which are not obtainable in the real drive, and the sensorless drive system loses stability. The stator currents reach very high values, which are not desirable in sensorless IM drives. It is worth to notice that for AFO and MRAS ${ }^{c C}$ with stability improvement method and MRAS ${ }^{\mathrm{CV}}$ the operation of the drive becomes excellent (Fig. 8 rows II, IV, V). Stator currents are limited, rotor speed and rotor flux coincide with their reference values.

\section{Conclusion}

A detailed stability analysis of the AFO, MRAS ${ }^{\mathrm{CC}}$ and MRAS ${ }^{\mathrm{cV}}$ speed estimators allows to unambiguously state that the MRAS ${ }^{\mathrm{CV}}$ estimator is characterized by stable operation in both the motoring and regenerating modes, in the case of properly identified IM equivalent circuit parameters. It seems to be the only one MRAS-type IM speed estimator, which does not lose stability in the regenerating mode. However, the use of an appropriate method to improve stability for the AFO and MRAS ${ }^{c C}$ estimators allows to achieve the same characteristics of all estimators in the case of theoretical analysis and simulation results. The characteristic feature of the AFO and MRAS ${ }^{\mathrm{CC}}$ estimators with the stability improvement method (equation 16) is the requirement to correct the angle $\varphi$ during the IM operation mode changes; it means to switch the angle value from (33) to 0 , when drive system changes the regenerating operation to motoring and vice versa. Experimental studies show that an important drawback of the MRAS ${ }^{\mathrm{cV}}$ estimator is the appearance of fixed angular velocity errors as the load torque increases. This disadvantage of MRAS ${ }^{\mathrm{CV}}$ is 
SPEED
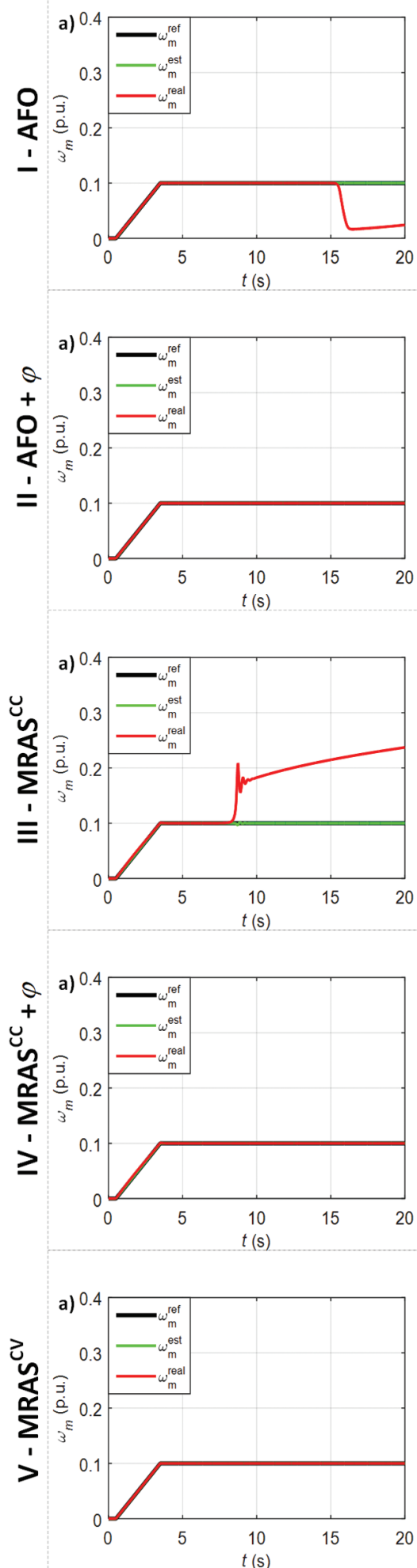

ROTOR FLUX
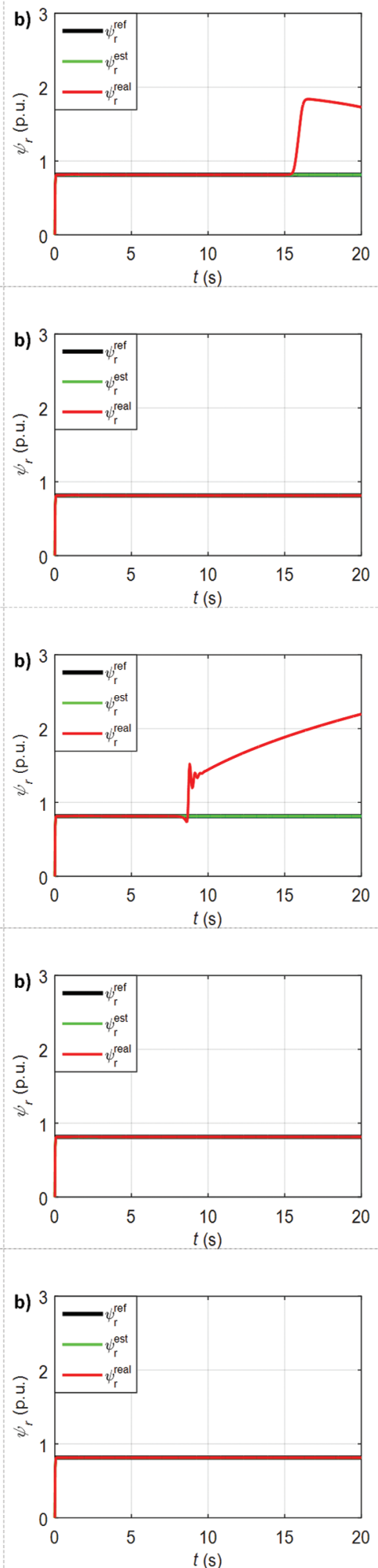

PHASE CURRENT
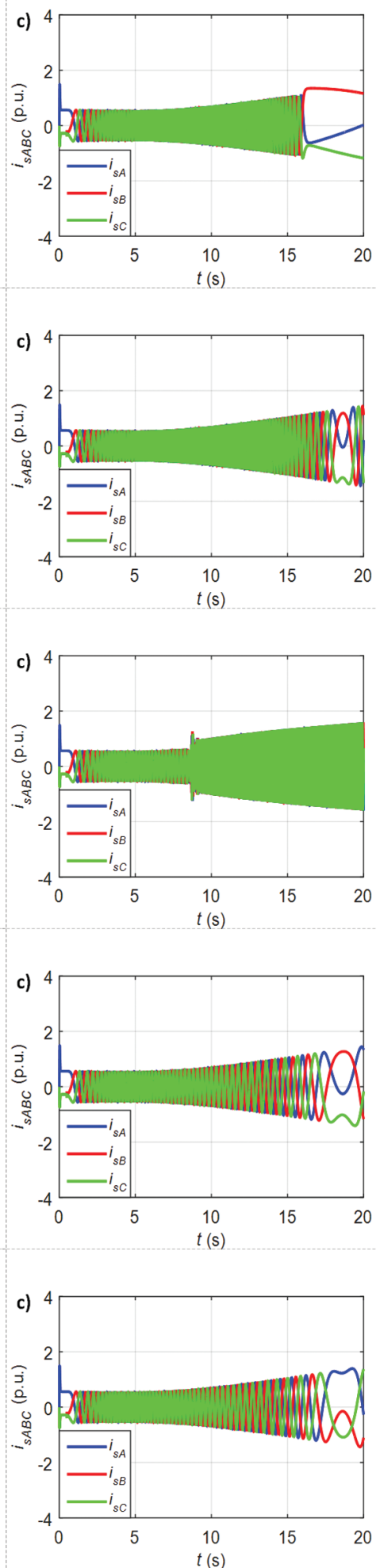

Fig. 8. Transients of the drive state variables under closed-loop operation of the estimators - simulation results. Column a) real, reference and estimated speed, column b) real, reference and estimated rotor flux, column c) stator currents 
connected with a significant impact of motor parameter mismatch on the estimation quality of this estimator. The abovementioned properties mean that, under real conditions, when motor parameters change due to variable operating points, the AFO and MRAS ${ }^{\mathrm{cC}}$ with proper method of stability improvement can behave better than the MRAS $^{\mathrm{CV}}$ estimator. However, authors plan to focus on solving this problem in future studies.

\section{Appendix}

Matrix $\Delta \hat{\mathbf{A}}_{0}^{e}$ has the following form:

$$
\Delta \hat{\mathbf{A}}_{0}^{A F O}=\Delta \hat{\mathbf{A}}_{0}^{C C}=\left[\begin{array}{ccc}
-j \Delta \omega_{s} & -j \frac{k_{r}}{l_{\sigma}} \Delta \omega_{m} & 0 \\
0 & -j \Delta \omega_{s}+j \Delta \omega_{m} & 0 \\
0 & 0 & 0
\end{array}\right], \Delta \hat{\mathbf{A}}_{0}^{C V}=\left[\begin{array}{ccc}
-j \Delta \omega_{s} & -j \frac{k_{r}}{l_{\sigma}} \Delta \omega_{m} & 0 \\
0 & -j \Delta \omega_{s} & 0 \\
0 & 0 & 0
\end{array}\right] .
$$

Coefficients $a_{51}-a_{55}$ are given as follows:

$$
\begin{gathered}
a_{51}=-\psi_{r e f} \sin \varphi\left[K_{i}-K_{p}\left(\frac{r_{1}}{l_{\sigma}}+g_{s x}\right)\right]-K_{p} \psi_{r e f}\left(\omega_{s 0}+g_{s y}\right) \cos \varphi \\
a_{52}=\psi_{r e f} \cos \varphi\left[K_{i}-K_{p}\left(\frac{r_{1}}{l_{\sigma}}+g_{s x}\right)\right]-K_{p} \psi_{r e f}\left(\omega_{s 0}+g_{s y}\right) \sin \varphi \\
a_{53}=-\frac{K_{p} \psi_{r e f} k_{r} \omega_{m 0} \cos \varphi}{l_{\sigma}}-\frac{K_{p} \psi_{r e f} k_{r} \sin \varphi}{\tau_{r} l_{\sigma}} \\
a_{54}=\frac{K_{p} \psi_{r e f} k_{r} \cos \varphi}{\tau_{r} l_{\sigma}}-\frac{K_{p} \psi_{r e f} k_{r} \omega_{m 0} \sin \varphi}{l_{\sigma}} \\
a_{55}=-\frac{K_{p} \psi_{r e f}^{2} k_{r} \cos \varphi}{l_{\sigma}}
\end{gathered}
$$

\section{Acknowledgments}

This work was supported by the National Science Centre Poland, under project 2015/17/B/ST7/03846 and partly by the statutory founds of the Department of Electrical Machines, Drives and Measurements, Wroclaw University of Science and Technology (2018).

\section{References}

Abu-Rub, H. and Oikonomou, N. (2008). Sensorless observer system for induction motor control. In: Proceedings of the IEEE Power Electronics Specialists Conference, Rhodes, 15-19 June 2008, pp. 30-36.

Barut, M., Bogosyan, S. and Gokasan, M. (2007). Speed-Sensorless Estimation for Induction Motors Using Extended Kalman Filters. IEEE Transactions on Industrial Electronics, 54(1), pp. 272-280.
Bensiali, N., Etien, E. and Benalia, N. (2015). Convergence Analysis of Back-EMF MRAS Observers Used in Sensorless Control of Induction Motor Drives. Mathematics and Computers in Simulation, 115, pp. 12-23.

Boldea, I., Lascu, C. and Blaabjerg, F. (2009). A Class of Speed-Sensorless Sliding-Mode Observers for High-Performance Induction Motor Drives. IEEE Transactions on Industrial Electronics, 56(2), pp. 3394-3403. 
Chen, B., Wang, T., Yao, W., Lee, K. and Lu, Z. (2014). Speed Convergence Rate-Based Feedback Gains Design of Adaptive Full-Order Observer in Sensorless Induction Motor Drives. IET Electric Power Applications, 8(1), pp. 13-22.

Dybkowski, M. and Orłowska-Kowalska, T. (2008). Application of the stator current-based MRAS speed estimator in the sensorless induction motor drive. In: 13th International Power Electronics and Motion Control Conference, EPE-PEMC, Poznan pp, 1-3 September 2008, pp. 2306-2311.

Etien, E., Chainge, C. and Bensiali, N. (2010). On the Stability of Full Adaptive Observer for Induction Motor in Regenerating Mode. IEEE Transactions on Industrial Electronics, 57(5), pp. 1599-1608.

Finch, J. W. and Giaouris D. (2008). Controlled AC Electrical Drives. IEEE Transactions on Industrial Electronics, 55(2), pp. 481-491.

Harnefors, L. and Hinkkanen, M. (2008). Complete Stability of Reduced-Order and Full-Order Observers for Sensorless IM Drives. IEEE Transactions on Industrial Electronics, 55(30), pp. 1319-1329.

Hinkkanen, M. and Luomi, J. (2004). Stabilization of Regenerating-Mode Operation in Sensorless Induction Motor Drives by Full-Order Flux Observer Design. IEEE Transactions on Industrial Electronics, 51(6), pp. 1318-1328.

Holtz, J. (2006). Sensorless Control of Induction Machines - With or Without Signal Injection?, IEEE Transactions on Industrial Electronics, 53(1), pp. 7-30.

Kazmierkowski, M. P. Blaabjerg, F., Kirshnan, R. (2002). Control in Power Electronic - Selected Problems. USA: Academic Press.

Korzonek, M. and Orłowska-Kowalska, T. (2016). Stability Analysis of MRASCC Speed Estimator in Motoring and Regenerating Mode. Power Electronics and Drives, 1(36) (2), pp. 113-131.

Korzonek, M. and Orłowska-Kowalska, T. (2017a). Dependence of the adaptive full-order observer stability on parameter identification errors. In: 2017 IEEE 26th International Symposium on Industrial Electronics (ISIE), Edinburgh, UK, 19-21 June 2017, pp. 277-283.

Korzonek, M. and Orłowska-Kowalska, T. (2017b). Influence of parameters identification errors on the stability boundaries of the modified MRAScc speed estimator of the induction motor drive. In: 2017 19th European Conference on Power Electronics and Applications (EPE'17 ECCE Europe), Warsaw, Poland, 11-14 September 2017, pp. 1-10.
Korzonek, M., Orłowska-Kowalska, T. and Tarchała, G. (2017). Stability Analysis of Selected Speed Estimators for Induction Motor Drive in Regenerating Mode: A Comparative Study. IEEE Transactions on Industrial Electronics, 64(10), pp. 7721-7730.

Kubota, H., Matsuse, K. and Nakano, T. (1993). DSPBased Speed Adaptive Flux Observer of Induction Motor. IEEE Transactions on Industry Applications, 29(2), pp. 344-348.

Kubota, H., Satao, I., Tamura Y., Matsuse, K., Ohta, H. and Hori, Y. (2002). Regenerating-Mode Low-Speed Operation of Sensorless Induction Motor Drive with Adaptive Observer. IEEE Transactions on Industry Applications, 38(4), pp. 1081-1086.

Kumar, R., Das, S., Syam, P. and Chattopadhyay, A. K. (2015). Review on Model Reference Adaptive System for Sensorless Vector Control of Induction Motor Drives. IET Electric Power Applications, 9(7), pp. 496-511.

Orłowska-Kowalska, T. and Dybkowski, M. (2010). Stator-Current-Based MRAS Estimator for A Wide Range Speed-Sensorless Induction-Motor Drive. IEEE Transactions on Industrial Electronics, 57(4), pp. 1296-1308.

Pacas, M. (2011). Sensorless Drives in Industrial Applications. IEEE Industrial Electronics Magazine, 5(2), pp. 16-23.

Pal, A., Das, S. and Chattopadhyay, A. K. (in press). An Improved Rotor Flux Space Vector Based MRAS for Field Oriented Control of Induction Motor Drives. IEEE Transactions on Power Electronics, doi: 10.1109/TPEL.2017.2657648.

Peng, F. Z. and Fukao, T. (1994). Robust Speed Identification for Speed-Sensorless Vector Control of Induction Motors. IEEE Transactions on Industry Applications, 30(5), pp. 1234-1240.

Purti, S. J., Kumar, R. and Das, S. (2015). Performance assessment of rotor flux and reactive power based MRAS for speed sensorless induction motor drive in a common test rig. In: 2015 International Conference of Computer, Communication and Control (IC4), Indore, India, 10-12 September 2015, pp. 1-7.

Rashed, M., Stronach, F. and Vas, P. (2003). A stable MRAS-based sensorless vector control induction motor drive at low speeds. In: IEEE International Electric Machines and Drives Conference, Madison, Vol. 1, 1-4 June 2003, pp. 139-144.

Schauder, C. (1992). Adaptive Speed Identification for Vector Control of Induction Motors without Rotational Transducers. IEEE Transactions on Industry Applications, 28(5), pp. 1054-1061 
Sobczuk, D. (1999). Application of ANN for Control of PWM Inverter Fed Induction Motor Drives. PhD dissertation, Warsaw University of Technology.

Stoicuta, O. and Pana, T. (2016). Asymptotic stability study of induction motor sensorless vector control systems with MRAS observer. In: 2016 IEEE International Conference on Automation, Quality and Testing, Robotics (AQTR), Cluj-Napoca, Romania, 19-21 May 2016, pp. 1-6.

Sunwankawin, S. and Sangwongwanich, S. (2006). Design Strategy of An Adaptive Full-Order Observer for Speed-Sensorless Induction Motor Drive - Tracking Performance and Stabilization. IEEE Transactions on Industrial Electronics, 53(1), pp. 96-119.

Tarchała, G., Orlowska-Kowalska, T. and Dybkowski, M. (2014). Sliding-Mode Direct Torque Control and Sliding-Mode Observer with A Magnetizing Reactance Estimator for the Field-Weakening of the Induction Motor Drive. Mathematics and Computers in Simulation, 98, pp. 31-45.

Vas, P. (1998). Sensorless Vector and Direct Torque Control. New York: Oxford University Press.

Verma, V. and Chakraborty, Ch. (2014). New series of MRAS for speed estimation of vector controlled induction motor drive. In: Proceedings of the 40th Annual Conference IEEE Industrial Electronics Society (IECON), Dallas, 29 October-1 November 2014, pp. 755-761.

Vonkomer, J. and Zalman, M. (2013). On the stability of current based MRAS. In: Proceedings of the 39th Annual Conference on IEEE Industrial Electronics Society (IECON), pp. 3018-3023.

Zaky, M. S. (2011). A Stable Adaptive Flux Observer for A Very Low Speed-Sensorless Induction Motor Drives Insensitive to Stator Resistance Variations. Ain Shams Engineering Journal, 2(1), pp. 11-20. 